Crescendo, Novartis, Genentech, Janssen, and AbbVie, cassandra calabrese Consultant of: Abbvie

GSK, Speakers bureau: Sanofi-Genzyme

DOI: 10.1136/annrheumdis-2020-eular.772

\section{FRI0495 \\ FOLLOW UP OF INTERSTITIAL PNEUMONIA WITH AUTOIMMUNE FEATURES - THE EXPERIENCE OF ONE CENTRE}

N. Madeira ${ }^{1}$, M. Alvarenga Santos ${ }^{2}$, L. Cunha Miranda ${ }^{3}$, S. Clemente ${ }^{2}$, S. Furtado ${ }^{2} .{ }^{1}$ Instituto Português de Reumatologia, Rheumatology, Lisbonne, Portugal; ${ }^{2}$ Hospital Beatriz Ângelo, Pulmonology, Loures, Portugal; ${ }^{3}$ Instituto Português de Reumatologia, Lisbonne, Portugal

Background: Interstitial Lung Diseases (ILD) may present features suggesting an underlying autoimmune process, which seem to differentiate them from idiopathic interstitial pneumonias, although without fully meeting the classification criteria (CC) for a specific connective tissue disease. Different terms had been used to describe these conditions and, to reach a consensus, the European Respiratory Society/American Thoracic Society proposed the CC for an entity named Interstitial Pneumonia with Autoimmune Features (IPAF). Clinical evolution and prognosis of this entity are still poorly understood.

Objectives: To evaluate clinical evolution and prognosis of a population of patients with IPAF.

Methods: Retrospective analysis of clinical files of patients followed by the Pulmonology Department since 02/2012 until 06/2019, who met the CC for IPAF, regarding clinical, functional and radiological evolution. Patients were considered to have a progressive phenotype in $24 \pm 3$ months from their $1^{\text {st }}$ evaluation if they fulfil 1 of the 4 criteria: relative decline in FVC $\geq 10 \%$ predicted; relative decline in $\mathrm{FVC} \geq 5-<10 \%$ predicted and worsened respiratory symptoms; relative decline in FVC $\geq 5-<10 \%$ predicted and increased extent of fibrosis on High-resolution Computed Tomography (HRCT); worsened respiratory symptoms and increased extent of fibrosis on HRCT.

Results: 22 (7.4\%) of 296 ILD patients met IPAF CC. $59.0 \%$ were female with an age at the $1^{\text {st }}$ evaluation of $66.7 \pm 12.4$ years. They were all non-smokers $(63.6 \%)$ or ex-smokers (36.4\%). Serologic and morphologic criteria were both present in $21(95.4 \%)$ and clinical criteria in 5 patients $(22.7 \%)$. Antinuclear antibodies (ANA) were identified in 19, rheumatoid factor in 4, SSA in 3 and anti-Jo-1 in 1 patient. HRCT patterns were identified in 21 patients: 15 nonspecific interstitial pneumonia (NSIP), 5 organizing pneumonia (OP) and 2 lymphocytic interstitial pneumonia (LIP). One NSIP and 1 LIP identified on HRCT were confirmed by histopathology. Three patients had inflammatory arthritis and 2 had Raynaud's phenomenon. Immunosuppressive therapy was introduced in most cases (18 patients, including systemic corticotherapy in 17, azathioprine in 4, mycophenolate mofetil in 1), azithromycin was prescribed in 2 patients and 3 remained without therapy. Regarding the follow up at $24 \pm 3$ months from the $1^{\text {st }}$ evaluation (3 patients were excluded due to too recent follow-up), 4 patients (18.2\%) had progressive phenotype, 7 (31.8\%) had a favourable evolution and $3(13.6 \%)$ patients had died. During a follow-up of $31.1 \pm 19.8$ months, this number rose to 6 patients $(27.3 \%)$, all of them died by respiratory cause and had NSIP pattern. No differences were found in age, last FVC, therapy and time of disease evolution between those who died and the others.

Conclusion: Our study showed that a small proportion of IPAF patients had a progressive phenotype and the NSIP pattern seemed to be a poor prognosis factor for survival.

References:

[1] Ito $Y$, Arita M, Kumagai S, et al. Serological and morphological prognostic factors in patients with interstitial pneumonia with autoimmune features. BMC Pulm Med 2017; 17:111 10.1186/s12890-017-0453-z

Disclosure of Interests: None declared

DOI: 10.1136/annrheumdis-2020-eular.4395

\section{FRI0496 $\quad$ FRECUENCY OF POLYAUTOIMMUNITY IN RHEUMATOID ARTHRITIS AND SYSTEMIC LUPUS ERITHEMATOSUS}

M. D. C. Ordoñez Cañizares ${ }^{1}$, N. Mena-Vázquez ${ }^{1}$, R. Redondo ${ }^{1}, \mathrm{~S}$. Manrique Arija $^{1}$, I. Ureña ${ }^{1}$, A. Fernandez-Nebro' ${ }^{1}$ Servicio de Reumatología del Hospital Regional Universitario de Málaga (HRUM). Instituto de Investigación Biomédica de Málaga (IBIMA), Málaga, Spain

Background:

Objectives: To study the frequency of polyautoinmunity and multiple autoinmune syndrome (MAS) in patients with rheumatoid arthritis (RA) and systemic lupus erithematosus (SLE).

Methods: Study design: We performed a cross-sectional study in patients with RA and SLE, and compared them with healthy subjects. Cases: RA patients classified by ACR/EULAR 2010 criteria and SLE patients classified by ACR/EULAR 2019 criteria. SLE and RA patients were compiled consecutively from a rheumatology clinic of the Regional University hospital of Malaga. Controls: subjects without rheumatologic autoimmune disease (AD) from the same population area. Protocol: All subjects filled out a predesigned questionnaire for the collection of polyautoimmunity data on the cutoff date. Main variables: polyautoimmunity was defined as co-occurrence of SLE or RA and other AD. Secondary variables: Rheumatologic, cutaneous, endocrine, digestive and neurological AD. MAS was defined as presence of three or more AD. Family history of SLE, RA and other autoimmune diseases were also collected. Statistic analysis: descriptive analysis, bivariate analysis and multivariable analysis were done. (Dependent variable: Polyautoimmunity).

Results: We recruited 109 patients with RA, 105 with SLE and 88 controls. Fifteen patients with RA (13.8\%), 43 with SLE $(41 \%)$ and 2 controls $(2.2 \%)$ reported polyautoimmunity. Table 1 describes the epidemiological characteristics, comor bidities and polyautoimmunity in study population. The most frequent AD associated with RA was Sjögren's syndrome (SS) (53.3\%) and SS (55.8\%) followed by the antiphospholipid syndrome (30.2\%) were associated with SLE. Hashimoto's thyroiditis and psoriasis were the next most frequent $A D$. According to family history, 5 patients with RA (33.3\%) and 12 with SLE (27.9\%) had a family history of first degree of other $\mathrm{AD}$. Obesity was associated with polyautoimmunity in $\mathrm{RA}(\mathrm{OR}=3,362, \mathrm{p}=0.034)$. In SLE, joint damage $(\mathrm{OR}=2.282, \mathrm{p}=0.038$ ) and anti-RNP antibodies $(O R=5.095, p=0.028$ ) were factors associated with polyautoimmunity and taking hydroxychloroquine was a protective factor $(\mathrm{OR}=$ $0.190, p=0.004)$

Conclusion: Polyautoimmunity in RA and especially in SLE is frequent. It was associated with obesity in RA and in SLE with joint damage and anti-RNP antibodies. The hydroxychloroquine appeared as a protective factor

\begin{tabular}{lcccc}
\hline Variables & $\begin{array}{c}\mathrm{RA} \\
(\mathrm{N}=109)\end{array}$ & $\begin{array}{c}\text { SLE } \\
(\mathrm{N}=105)\end{array}$ & $\begin{array}{c}\text { Controls } \\
(\mathrm{N}=88)\end{array}$ & P value \\
\hline Epidemiological characteristics & & & & \\
Sex: female, $\mathrm{n}(\%)$ & $85(78.0)$ & $99(94.3)$ & $68(77.3)$ & 0.001 \\
Age, mean (SD), years & $56.5(10.8)$ & $50.8(13.2)$ & $57.1(10.6)$ & 0.133 \\
Caucasic race, $\mathrm{n}(\%)$ & $107(98.2)$ & $105(100)$ & $88(100)$ & 0.168 \\
Comorbidities & & & & \\
Smoking & & & & 0.001 \\
$\quad$ No smoking, $\mathrm{n}(\%)$ & $55(50.5)$ & $78(74.3)$ & $56(63.6)$ & \\
$\quad$ Smoking history, $\mathrm{n}(\%)$ & $54(49.5)$ & $27(25.7)$ & $32(36.4)$ & \\
Obesity, $\mathrm{n}(\%)$ & $38(34.9)$ & $21(20.0)$ & $22(25.0)$ & 0.044 \\
Dyslipidemia, $\mathrm{n}(\%)$ & $24(22.0)$ & $22(21.2)$ & $17(19.3)$ & 0.896 \\
Hypertension, $\mathrm{n}(\%)$ & $27(24.8)$ & $28(26.7)$ & $23(26.1)$ & 0.746 \\
Diabetes mellitus, $\mathrm{n}(\%)$ & $7(6.4)$ & $1(1.0)$ & $1(1.1)$ & 0.031 \\
Polyautoimmunity, $\mathrm{n}(\%)$ & $15(13.8)$ & $43(41)$ & $2(2.2)$ & $<0.001$ \\
MAS, $\mathrm{n}(\%)$ & $1(0.9)$ & $9(8,6)$ & $0(0.0)$ & $<0.001$ \\
FH polyautoimmunity, n (\%) & $19(17.6)$ & $26(24.8)$ & $15(17.0)$ & 0.060 \\
\hline
\end{tabular}

RA: rheumatoid arthritis; SLE: systemic lupus erithematosus; SD: standard desviation; MAS: multiple autoinmune syndrome; FH:Family history

Disclosure of Interests: None declared

DOI: 10.1136/annrheumdis-2020-eular.3105

\begin{tabular}{|l|l|}
\hline FRI0497 & THERAPEUTIC STRATEGIES AND LONG-TERM \\
OUTCOME IN PATIENTS WITH INTERSTITIAL \\
PNEUMONIA WITH AUTOIMMUNE FEATURES: A \\
SINGLE CENTER LARGE-SCALE OBSERVATIONAL \\
COHORT STUDY
\end{tabular}

O. Murata ${ }^{1,2}$, K. Suzuki $^{1}$, N. Sasaki ${ }^{2}$, T. Takeuchi ${ }^{1}$, M. Maemondo ${ }^{2}$. ${ }^{1}$ Keio University School of Medicine, Division of Rheumatology, Department of Internal Medicine, Tokyo, Japan; ${ }^{2}$ Iwate Medical University School of Medicine, Division of Pulmonary medicine, Allergy, and Rheumatology, Department of Internal Medicine, Morioka, Japan

Background: Patients with idiopathic interstitial pneumonia (IIP) may have features of connective tissue diseases (CTDs). The term interstitial pneumonia with autoimmune features (IPAF) has been recently proposed for such patients [1]. To date, only few studies have comprehensively described outcomes over a longterm period and choices of treatment [2-4].

Objectives: The aim of this study was to investigate the therapeutic strategies and long-term outcome among patients with IPAF, IIP, and CTD-ILD.

Methods: Six hundreds- and seventy-two patients who had visited ou department between April 2009 and March 2019 and were evaluated by chest HRCT scan. They were clinically and radiologically diagnosed as having interstitial lung disease (ILD), including IIP, CTD-ILD, undifferentiated connective tissue diseases associated ILD or other ILD. Then, we applied IPAF criteria to these patients, 68 patients were diagnosed as IPAF. We extracted the 